\title{
Prevalence of Cognitive Impairment in Older Adults with Heart Failure
}

\author{
Tanya R. Gure, MD, ${ }^{a}$ Caroline S. Blaum, MD, MS, ${ }^{a b}$ Bruno Giordani, PhD, ${ }^{c}$ Todd M. Koelling, \\ $M D,{ }^{d}$ Andrzej Galecki, MD, PhD, ${ }^{\text {aef }}$ Susan J. Pressler, PhD, ${ }^{g}$ Scott L. Hummel, MD, MS, ${ }^{d h}$ and \\ Kenneth M. Langa, MD, PhD ${ }^{i j k}$
}

OBJECTIVES: To determine the prevalence of cognitive impairment in older adults with heart failure (HF).

DESIGN: Cross-sectional analysis of the 2004 wave of the nationally representative Health and Retirement Study linked to 2002 to 2004 Medicare administrative claims.

SETTING: United States, community.

PARTICIPANTS: Six thousand one hundred eighty-nine individuals aged 67 and older.

MEASUREMENTS: An algorithm was developed using a combination of self- and proxy report of a heart problem and the presence of one or more Medicare claims in administrative files using standard HF diagnostic codes. On the basis of the algorithm, three categories were created to characterize the likelihood of a HF diagnosis: high or moderate probability of HF, low probability of $\mathrm{HF}$, and no HF. Cognitive function was assessed using a screening measure of cognitive function or according to proxy rating. Age-adjusted prevalence estimates of cognitive impairment were calculated for the three groups.

RESULTS: The prevalence of cognitive impairment consistent with dementia in older adults with HF was $15 \%$, and the prevalence of mild cognitive impairment was $24 \%$. The odds of dementia in those with HF were significantly higher, even after adjustment for age, education level, net

From the ${ }^{\mathrm{a}}$ Division of Geriatric and Palliative Medicine, Department of Internal Medicine, University of Michigan, ${ }^{\mathrm{b}}$ Geriatric Research, Clinical and Education Center, Veterans Affairs Ann Arbor Healthcare System, ${ }^{\mathrm{c}}$ Department of Psychiatry, University of Michigan, ${ }^{\mathrm{d}}$ Division of Cardiovascular Medicine, Department of Internal Medicine, University of Michigan, ${ }^{\mathrm{e}}$ Department of Biostatistics, University of Michigan, ${ }^{\mathrm{f}}$ Institute of Gerontology, University of Michigan, ${ }^{\mathrm{g}} \mathrm{S}$ chool of Nursing, University of Michigan, ${ }^{\mathrm{h} D i v i s i o n}$ of Cardiology, Veterans Affairs Ann Arbor

Healthcare System, ${ }^{\mathrm{i} D i v i s i o n}$ of General Internal Medicine, Department of Internal Medicine, University of Michigan, ${ }^{\mathrm{j} C e n t e r}$ for Practice Management and Outcomes Research, Veterans Affairs Ann Arbor Healthcare System, and ${ }^{\mathrm{k}}$ Institute for Social Research, University of Michigan, Ann Arbor, Michigan.

Address correspondence to Tanya R. Gure, Division of Geriatric and Palliative Medicine, Department of Internal Medicine, University of Michigan, 300 North Ingalls Bldg., Rm. 901, Ann Arbor, MI 48109.

E-mail: tanruff@umich.edu

DOI: 10.1111/j.1532-5415.2012.04097.x worth, and prior stroke (odds ratio $=1.52,95 \%$ confidence interval $=1.14-2.02$ ).

CONCLUSION: Cognitive impairment is common in older adults with $\mathrm{HF}$ and is independently associated with risk of dementia. A cognitive assessment should be routinely incorporated into HF-focused models of care. J Am Geriatr Soc 60:1724-1729, 2012.

Key words: heart failure; aged; cognitive impairment; Medicare claims data

$\mathrm{H}_{\mathrm{i}}$ eart failure (HF) predominantly affects older persons, in whom multiple complex chronic conditions often coexist. ${ }^{1-4} \mathrm{HF}$ remains the leading cause of hospitalization in adults aged 65 and older in the United States. Several initiatives exist to reduce the mortality, morbidity, and healthcare utilization of older adults with HF, but these recent innovations in HF management have generally not translated into lower mortality and rehospitalization rates. ${ }^{1,5,6}$ The reasons for unchanged outcomes may stem from the lack of attention given to prognostically important conditions, such as cognitive impairment, ${ }^{7,8}$ yet understanding of the prevalence of cognitive impairment in older adults with HF is limited. Individuals with HF who have mild cognitive impairment (MCI) or dementia may have a greater risk of rehospitalization and mortality because of poor adherence to prescribed therapy due to the complexity of self-management tasks in HF management. $^{9-11}$

Cognitive impairment, including dementia and MCI, is associated with cerebrovascular and cardiovascular disease and is linked to $\mathrm{HF},{ }^{6,12,13}$ but its prevalence and effects on morbidity and mortality are not well understood in individuals with HF. Prevalence estimates for cognitive impairment in individuals with HF vary widely, from 5\% to $75 \%$, with most estimates falling between $25 \%$ and $46 \% .^{12-15}$ Prior work examining the association between 
HF and cognitive impairment has consisted of a casecontrol study design using nonrepresentative samples, including inpatients and those with a range of etiologies of HF. Furthermore, the assessment of cognition has not been standardized; investigators have used a myriad of tools, ranging from cognitive screens such as the Mini-Mental State Examination to abbreviated neuropsychological test batteries. ${ }^{15-17}$ Because of these and other limitations, it is difficult to confidently assess the true extent of cognitive impairment in older adults with HF. The research hypotheses of the current study were that older adults with HF would have a higher prevalence of cognitive impairment than other individuals with heart conditions and that HF would be independently associated with a higher risk of cognitive impairment.

The goal was to determine the prevalence of cognitive impairment in older adults with HF using a nationally representative sample and to determine whether HF is independently associated with cognitive impairment.

\section{METHODS}

\section{Data}

Data were used from the 2004 wave of the Health and Retirement Study (HRS), a biennial, longitudinal survey of a nationally representative cohort of U.S. adults aged 51 and older. ${ }^{18}$ The HRS provides detailed self-report information on chronic diseases and task-specific disabilities.

\section{Sample Definition}

Ninety percent of HRS respondents provided consent to link their Medicare claims to their HRS survey data. Claims data from 2 years before the 2004 interview were viewed to capture the greatest number of HRS respondents with HF in the Medicare claims data. ${ }^{19,20}$ In the 2004 survey wave, 9,663 respondents were aged 67 and older, ensuring that each individual had 2 full years of Medicare eligibility during the surveillance period. The sample was further reduced to 8,207 after exclusion of HRS respondents who were not continuously enrolled in Medicare Parts $\mathrm{A}$ and $\mathrm{B}$, and 1,650 respondents enrolled in Medicare managed care were excluded because these plans may not have complete data. Finally, 429 respondents in nursing homes at the time of the analysis were excluded. Thus, the analytical sample included 6,189 respondents, representative of approximately 23.4 million communitydwelling adults aged 67 and older with Medicare feefor-service in the United States in 2004.

\section{Definition of HF and Other Heart Problems According to Self- or Proxy Report}

Self- or proxy report of heart problems, including HF, was determined based on survey responses to questions in the HRS 2004 core interview wave. All respondents and proxies were asked: "Has a doctor ever told you that you had a heart attack, coronary heart disease, angina, congestive heart failure, or other heart problems?" The response to this question was used to sort respondents into two mutually exclusive heart problem categories: no HF or other heart problem and heart problem (including $\mathrm{HF}$ ).

\section{Claims-Based Diagnosis of HF}

HF was defined using Medicare claims according to the International Classification of Diseases, Ninth Revision, Clinical Modification (ICD-9-CM) diagnosis codes for primary and secondary diagnoses. Using Medicare Part A and Part B claim files, the claims-based diagnosis of HF for the 2-year period before each respondent's HRS interview date was determined. ${ }^{19}$ ICD-9-CM coding algorithms were used to identify $\mathrm{HF}$ as a diagnosis in inpatient, outpatient, and carrier files using codes 398.91, 402.01 to 402.91 , 404.01 to 404.93 , and 428.0 to 428.90 over a 2 -year period. ${ }^{19}$

\section{HF Case-Finding Approach}

Prior work had identified the test-operating characteristics of self- or proxy report of HF by comparing self- or proxy report with HF diagnosis codes in linked Medicare claims as a diagnostic criterion standard (sensitivity, 25\%; specificity, 99\%). ${ }^{21}$ Older adults in the HRS were more likely to report HF if they had poorer health. Underreporting of HF was much more common in this study, in particular within minority populations and in individuals with fewer Medicare claims, perhaps suggesting less-severe disease. Therefore, the presence of a heart problem was used to better capture respondents who may have been unaware of $\mathrm{HF}$ as a diagnosis. A newly developed algorithm was derived from this work (Figure 1). A combination of self- and proxy report of a heart problem and the presence of one or more Medicare claims in inpatient, outpatient, and carrier files using standard HF diagnostic codes was used to increase the yield of HF cases. On the basis of the algorithm, three categories were created to characterize the likelihood of having a HF diagnosis: high to moderate probability of $\mathrm{HF}$, low probability of $\mathrm{HF}$, and no HF.

\section{Survey-Based Assessment of Cognitive Performance}

For self-respondents, the presence of dementia was assessed using a modified version of the Telephone Interview for Cognitive Status (TICS-m), a validated cognitive screening instrument patterned on the Mini-Mental State Examination. $^{22}$ The TICS-m used in the HRS is a 27-point scale and has high sensitivity and specificity for cognitive impairment and dementia in community samples of older adults. ${ }^{22-24}$ The test items include immediate and delayed 10 -word recall test to measure memory, serial seven subtraction test to measure working memory, and counting backward test to measure speed of mental processing. ${ }^{25,26}$ Cut-scores to identify individuals with cognitive impairment were based on findings from the Aging, Demographics and Memory Study. ${ }^{27}$ Based on prior work validating these cut-points, cognitive function was categorized as normal ( $\geq 12$ points) or impaired $(<12$ points), with the impaired category divided into mild (7-11 points) and moderate to severe impairment consistent with dementia (0-6 points). ${ }^{27}$ Cognitive categories for respondents with proxy informants were based on proxy and interviewer 


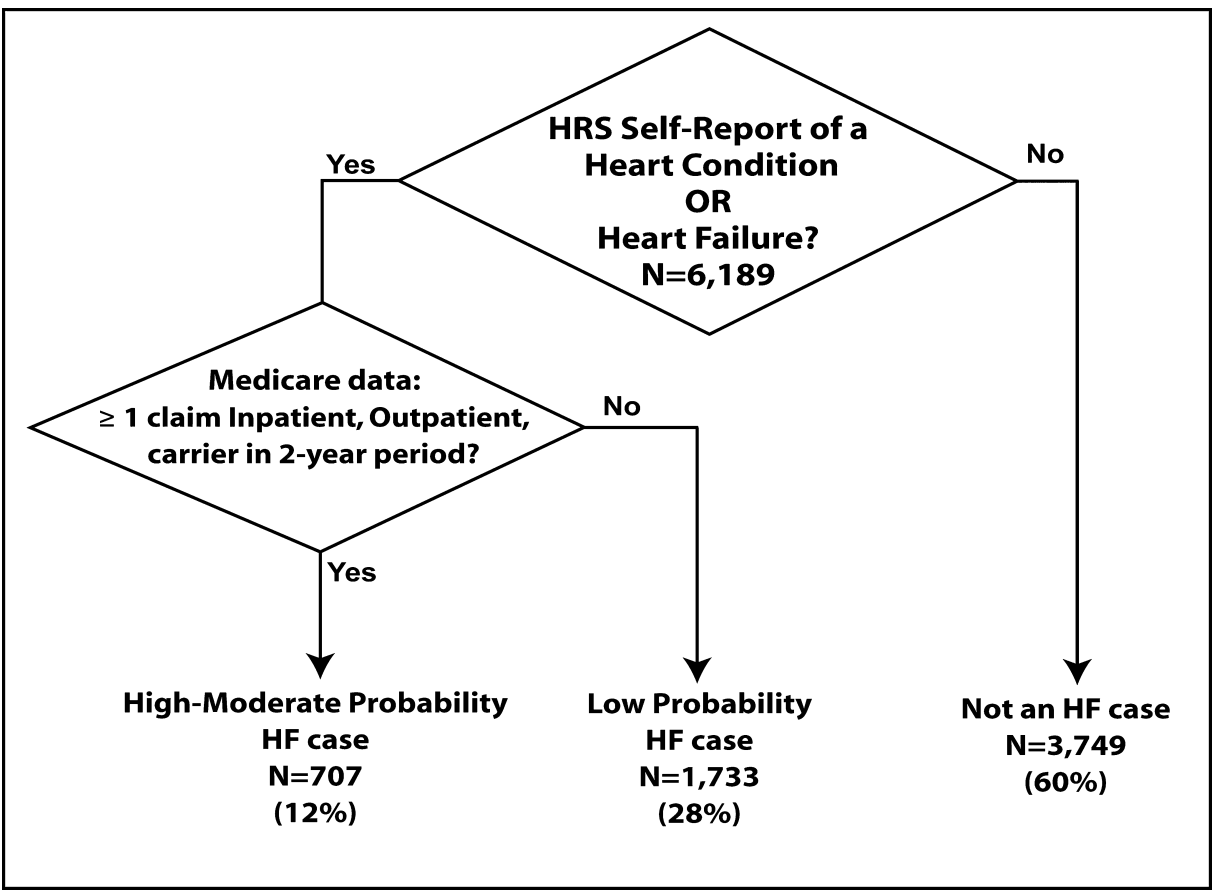

Figure 1. Ascertainment of heart failure cases in Health and Retirement Study and Medicare data using a newly developed algorithm, weighted $\%$ (95\% confidence interval). SD = standard deviation.

assessments of cognitive function. Proxies assessed memory and whether the respondent was cognitively impaired. Cognitive function scores were created based on proxy interview ratings and number of instrumental activity of daily living limitations, resulting in a scale ranging from 0 to 11 , with scores from 3 to 5 indicating MCI and scores from 6 to 11 indicating cognitive impairment consistent with dementia.

\section{Independent Variables}

The sociodemographic variables included in the analysis as independent variables were age, race, sex, household net worth, and years of education. Self-reported chronic conditions included were stroke and diabetes mellitus All measures were obtained from the HRS core interview in 2004.

\section{Data Analysis}

All analyses were conducted using STATA/SE 11 (StataCorp, College Station, TX). To adjust for the complex sample design of the HRS, the differential probability of selection, and the probability of a respondent's consent to accessing their Medicare data, all analyses were appropriately weighted. Analyses were conducted to calculate population-based characteristics and the prevalence of cognitive impairment based on sample data. Sociodemographic characteristics, TICS-m score, and proxy rating of cognitive status of the three heart condition categories were compared using adjusted Wald and Pearson chisquare test statistics as appropriate. Prevalence estimates of cognitive function were compared using categorical cognitive variables (normal, MCI, and moderate to severe cognitive impairment) using adjusted Wald chi-square statistical tests. Taylor series linearization was used to estimate design-based standard errors for population estimates (incorporating the stratification and clustering of the HRS sample along with the weights) and compute $95 \%$ confidence intervals (CIs) for population parameters.

Multivariate logistic regression was used to identify covariates independently associated with the likelihood of moderate to severe cognitive impairment according to heart disease category. The group with MCI were combined with the group with normal cognition for these analyses. The final logistic models included the sociodemographic and health status factors associated with cognitive impairment. Model 1 was a univariate analysis; Model 2 was adjusted for age only; and Model 3 was adjusted for age, race, educational level, net worth, and self-reported prior stroke.

\section{RESULTS}

\section{Sample Characteristics}

Of the 6,189 respondents in the analytical sample, the case-finding algorithm indicated that $707(11.7 \%$ of the population) had a moderate to high probability of HF. This corresponds to approximately 2,700,000 Medicare fee-for-service beneficiaries aged 67 and older with HF in the United States in 2004. The mean age was $76.5,42 \%$ were male, $8 \%$ were black, and $4 \%$ were Hispanic (Table 1). The mean number of years of education was 12.1. Five thousand seven hundred fifty-three (92\%) respondents were self-respondents, and $436(8 \%)$ were represented by a proxy informant.

The group with moderate to high probability of HF were older (mean age 78.6) than the groups with a low probability and no HF $(P<.001)$. The group with 
Table 1. Characteristics of Individuals Aged 67 and Older with Health and Retirement Study Data Linked to Medicare Claims

\begin{tabular}{|c|c|c|c|c|c|}
\hline Age mean (SD) & $75.6(6.51)$ & $77.4(6.75)$ & $78.6(6.86)$ & $76.5(6.71)$ & $<.001$ \\
\hline \multicolumn{6}{|l|}{ Race or ethnicity, $n$ (weighted \%) } \\
\hline Hispanic & $229(4)$ & $75(3)$ & $37(3)$ & $341(4)$ & .37 \\
\hline \multicolumn{6}{|l|}{ Sex, $\mathrm{n}$ (weighted \%) } \\
\hline Male & $1,414(38)$ & $828(48)$ & $334(47)$ & $2,576(42)$ & $<.001$ \\
\hline Female & $2,335(62)$ & $905(52)$ & $373(53)$ & $3,613(58)$ & \\
\hline Education, years, mean \pm SD & $12.3 \pm 3.2$ & $11.9 \pm 3.3$ & $11.4 \pm 3.3$ & $12.1 \pm 3.3$ & $<.001$ \\
\hline \multicolumn{6}{|l|}{ Net worth, \$ (quartile), n (weighted \%) } \\
\hline$\leq 61,000$ & $931(23)$ & $503(27)$ & $263(36)$ & $1,697(26)$ & $<.001$ \\
\hline Diabetes, $\mathrm{n}$ (weighted \%) & $593(15)$ & $418(24)$ & $234(.32)$ & $1,245(20)$ & $<.001$ \\
\hline \multicolumn{6}{|l|}{ Respondent, $\mathrm{n}$ (row \%, column \%) weighted $\mathrm{n}$} \\
\hline \multirow[t]{2}{*}{ Self } & $3,526(61,93)$ & $1,607(28,91)$ & $620(11,85)$ & $5,753(92)$ & $<.001$ \\
\hline & $13,000,000$ & $5,900,000$ & $2,300,000$ & $21,000,000$ & \\
\hline \multirow[t]{2}{*}{ Proxy } & $223(51,7)$ & $126(29,9)$ & $87(21,15)$ & $436(8)$ & \\
\hline & $1,000,000$ & 600,000 & 400,000 & $1,900,000$ & \\
\hline $\begin{array}{l}\text { Modified Telephone Interview for Cognitive } \\
\text { Status score, mean } \pm \text { SD (range } 0-27 \text { ) }\end{array}$ & $14.5 \pm 4.3$ & $13.7 \pm 4.6$ & $12.7 \pm 4.3$ & $14.1 \pm 4.4$ & $<.001$ \\
\hline $\begin{array}{l}\text { Proxy assessment of memory, } \\
\text { mean } \pm \text { SD (range } 0-11 \text { ) }\end{array}$ & $5.1 \pm 3.4$ & $5.0 \pm 3.3$ & $5.7 \pm 3.2$ & $5.2 \pm 3.3$ & .41 \\
\hline \multicolumn{6}{|c|}{ Cognitive category, $\mathrm{n}$ (weighted \%; $95 \%$ confidence interval) ${ }^{\mathrm{a}}$} \\
\hline Normal & $2,698(71 ; 70-73)$ & $1,126(68 ; 66-70)$ & $411(61 ; 57-65)$ & $4,235(69 ; 68-71)$ & $<.001$ \\
\hline
\end{tabular}

$\mathrm{HF}=$ heart failure; $\mathrm{SD}=$ standard deviation.

${ }^{a}$ Adjusted for age.

moderate to high probability of HF had a lower education level.

The majority of respondents in all heart condition categories were self-respondents, with $8 \%$ of respondents using a proxy informant. Twenty-percent of proxy informants had a high likelihood of HF, compared with $29 \%$ in the low-probability HF group and $51 \%$ in the no HF group. The mean TICS-m score for self-respondents was $14.1 \pm 4.4$. The group with a high to moderate probability of HF had the lowest mean TICS-m score $(12.7 \pm 4.3$; low probability HF, 13.7 ; no HF, $14.5 ; P<.001)$. There was no statistically significant difference in proxy rating score between the heart condition groups.

\section{Prevalence of Cognitive Impairment According to Heart Condition Category}

The age-adjusted cognitive performance of respondents with HRS Medicare data, is described in Table 1 according to heart condition category. The age-adjusted prevalence of moderate to severe cognitive impairment consistent with dementia in the group with the greatest probability of $\mathrm{HF}$ was $15 \% \quad(95 \% \mathrm{CI}=12-18 \%$; $P \leq .001$ compared with the groups with no HF and a low probability of $\mathrm{HF})$ and for MCI it was $24 \%(95 \%$ $\mathrm{CI}=21-28 \%)$. The age-adjusted prevalence of MCI was not significantly different between the different heart condition groups (Wald test $P=.11$ comparing the group with a low probability of HF with the group with no HF; $P=.34$ comparing the group with a low probability of HF with the group with a moderate to high probability of $\mathrm{HF})$.

\section{Association Between Cognitive Impairment and HF}

In a series of multivariate logistic regression models predicting the likelihood of moderate to severe cognitive impairment according to heart condition category, HF was consistently associated with significantly greater odds of moderate to severe cognitive impairment (Table 2). Age had the greatest effect size in the model, as expected, given its strong association with MCI and dementia. In the final model, the addition of race, educational level, net worth, and self-reported prior stroke further attenuated the effect of $\mathrm{HF}$ on dementia (odds ratio $(\mathrm{OR})=1.52,95 \%$ $\mathrm{CI}=1.14-2.02$ ). Sex and self-reported diabetes mellitus were not significant predictors of moderate to severe cognitive impairment in the final model. The group with low 
Table 2. Predictors of Moderate to Severe Cognitive Impairment, Logistic Regression $(\mathrm{N}=6,189)$

\begin{tabular}{|c|c|c|c|}
\hline $\begin{array}{c}\text { Heart } \\
\text { Condition }\end{array}$ & Univariate & Age Adjusted & $\begin{array}{l}\text { Multivariate } \\
\text { Adjustment }^{\mathrm{a}}\end{array}$ \\
\hline $\begin{array}{c}\text { (Reference } \\
\text { No HF) }\end{array}$ & \multicolumn{3}{|c|}{ Odds Ratio (95\% Confidence Interval) } \\
\hline $\begin{array}{l}\text { Low } \\
\text { probability } \\
\text { of HF }\end{array}$ & 1.55 (1.24-1.99) & $1.33(1.05-1.68)$ & $1.19(0.94-1.51)$ \\
\hline $\begin{array}{l}\text { Moderate } \\
\text { to high } \\
\text { probability } \\
\text { of HF }\end{array}$ & $2.50(1.99-3.14)$ & $1.95(1.50-2.54)$ & $1.52(1.14-2.02)$ \\
\hline
\end{tabular}

$\mathrm{HF}=$ heart failure.

a Covariates are age, race, educational level, net worth, and self-reported prior stroke.

probability of HF had greater odds of moderate to severe cognitive impairment when age was the only covariate in the model, although the odds were not significantly different from those of the group without HF in the full model $(\mathrm{OR}=1.19 ; 95 \% \mathrm{CI}=0.94-1.51)$.

\section{DISCUSSION}

In a unique nationally representative sample from the HRS Medicare claims linked data, it was found that approximately $40 \%$ of older adults aged 67 and older with HF are cognitively impaired, with $15 \%$ demonstrating moderate to severe impairment consistent with dementia. HF was independently associated with risk for cognitive impairment. The prevalence estimates of cognitive impairment fell within the range $(25-46 \%)$ that prior studies have most commonly reported, but the prevalence of moderate to severe cognitive impairment consistent with dementia was lower than previously reported. It was also found that HF has a significant, independent association with dementia after adjustment for important sociodemographic and clinical predictors.

Comparing prevalence estimates across studies is difficult because of differences in participant samples (in HF type and study setting) and the large variability in cognitive tests used across studies. The strengths of the current study are its use of a broad case-mix of HF cases by using an algorithm that probably captures the mild to severe stages of HF and the presence of a comparison group within the cohort and its use of standardized cognitive measures that are validated from clinically diagnosed cognitively impaired cases from the Aging, Demographics, and Memory Study, an epidemiological substudy of dementia in the HRS. ${ }^{26}$ The TICS-m and informant assessment of cognitive performance discriminate well between individuals with dementia and those with MCI. ${ }^{22}$

Several limitations of the study warrant mention. First, self-report and Medicare claims data have limited validity as accurate measurements of HF. Multiple factors such as illness severity, socioeconomic background, race, patient health awareness gained through encounters with healthcare providers, and effective self-management skills can influence an individual's awareness of a heart condition. The validity of Medicare administrative data is also highly dependent on many factors: ICD-9 coding practices, organizational culture, coder experience, and the type of administrative file used. ${ }^{28}$ For these reasons, the prevalence estimates may be an over- or underestimation of HF cases in the population. Using HRS Medicare linked data in prior work, self-reported prevalence of $\mathrm{HF}$ at $5 \%$ is an undercount and claims-based prevalence at $16 \%$, an overcount. ${ }^{21}$ Thus, the algorithm, which combines self- and proxy report of a heart problem with one or more Medicare diagnostic HF claims, is probably more accurate than use of self- or proxy report or claims data alone in this data source.

Second, medical records were not available to adjudicate cases further, ascertain HF severity, or determine the use of medications that might affect cognition. Third, approximately $11 \%$ of the HRS respondents did not consent to the linkage of their survey data to Medicare claims. These factors may have introduced selection bias because the population enrolled in managed care programs differed from that with traditional Medicare fee-for-service plans with respect to health and socioeconomic status. ${ }^{29}$ Fourth, the cognitive measures used may have insufficiently probed the cognitive domains most commonly affected in individuals with HF, leading to inaccuracies in the findings. The estimates of moderate to severe impairment are lower than in prior epidemiological reports, probably because of the exclusion of nursing home residents. ${ }^{30}$ Nonetheless, this work provides one of the first estimates of global cognitive function in a nationally representative sample.

Cognitive impairment commonly co-occurs with HF in older adults, yet assessing cognition is not routinely incorporated into traditional HF disease management models of care. Awareness of cognitive status by healthcare providers, in particular HF care providers, is important for setting expectations for patient participation in a HF treatment plan and achieving optimal care. The current study found an independent association between HF and the presence of moderate to severe cognitive impairment-a cognitive performance level most consistent with dementia. Cognitive impairment is an underrecognized comorbid condition in community-dwelling older adults with HF that has important effects on prognosis, physical function, quality of life, healthcare utilization, and mortality.

\section{ACKNOWLEDGMENTS}

The authors appreciate the assistance of Yuo-Yu Lee, MS, and Jinkyung $\mathrm{Ha}, \mathrm{PhD}$, with data management and statistical analysis in the completion of this work and the thoughtful input of the participating faculty in the Junior Faculty Career Development Writing Workshop, sponsored by the Division of Geriatric and Palliative Medicine at the University of Michigan. An earlier version of this work was presented at the National Pepper Center meeting, Washington, DC, April 11, 2011.

Conflict of Interest: Dr. Koelling received research funding from Medtronic, Inc. and MESPERE, Inc. and an honorarium $(<\$ 10,000)$ from Abbott Vascular. He also served as a pro bono expert witness in a myocardial infarction case. 
Author Contributions: Gure T.R.: study concept and design, data analysis and interpretation, manuscript preparation. Blaum C.S.: data analysis and interpretation, manuscript preparation. Giordani B., Koelling T.M., Galecki A., Pressler S.J., and Hummel S.L.: data interpretation and manuscript preparation. Langa K.M.: study concept and design, data interpretation, manuscript preparation.

Sponsor's Role: None.

Funding: The National Institute on Aging (NIA) provided funding for the HRS (U01 AG09740), which is performed at the Institute for Social Research, University of Michigan. Dr. Langa was supported by NIA Grant R01 AG030155. Dr. Blaum was supported by the Ann Arbor Veterans Affairs Geriatric Research, Education and Clinical Center. Dr. Hummel was supported by National Heart, Lung and Blood Institute Grant K23 HL109176. Dr. Gure was supported by NIA Diversity Supplement Award R01 AG027010-02S1, the John A. Hartford Foundation Center of Excellence, the NIA Claude D. Pepper Center Research Career Development Core, and the National Center for Research Resources (NCRR; UL1 RR024986). The content is solely the responsibility of the authors and does not necessarily represent the official views of NCRR or the National Institutes of Health.

\section{REFERENCES}

1. Jessup M, Abraham WT, Casey DE et al. 2009 Focused update: ACCF/ AHA Guidelines for the Diagnosis and Management of Heart Failure in Adults. A report of the American College of Cardiology Foundation/American Heart Association Task Force on Practice Guidelines. Circulation 2009;119:1977-2016.

2. Kitzman DW, Gardin JM, Gottdiener JS et al. Importance of heart failure with preserved systolic function in patients $>$ or $=65$ years of age. CHS Research Group. Cardiovascular Health Study. Am J Cardiol 2001;87:413419.

3. Gure TR, Kabeto MU, Blaum CS et al. Degree of disability and patterns of caregiving among older Americans with congestive heart failure. J Gen Intern Med 2008;23:70-76.

4. Braunstein JB, Anderson GF, Gerstenblith G et al. Noncardiac comorbidity increases preventable hospitalizations and mortality among Medicare beneficiaries with chronic heart failure. J Am Coll Cardiol 2003;42:1226-1233.

5. Jencks SF, Williams MV, Coleman EA. Rehospitalizations among patients in the Medicare fee-for-service program. N Engl J Med 2009;360:14181428.

6. Tilvis RS, Kahonen-Vare MH, Jolkkonen J et al. Predictors of cognitive decline and mortality of aged people over a 10 -year period. J Gerontol B Psychol Sci Soc Sci 2004;59B:268-274.

7. Chaudhry SI, Wang Y, Gill TM et al. Geriatric conditions and subsequent mortality in older patients with heart failure. J Am Coll Cardiol 2010;55:309-316.

8. Lee DS, Austin PC, Rouleau JL et al. Predicting mortality among patients hospitalized for heart failure-derivation and validation of a clinical model. JAMA 2003;290:2581-2587.

9. Pressler SJ, Kim J, Riley P et al. Memory dysfunction, psychomotor slowing, and decreased executive function predict mortality in patients with heart failure and low ejection fraction. J Card Fail 2010;16:750-760.
10. Horowitz C, Rein S, Leventhal H. A story of maladies, misconceptions and mishaps: Effective management of heart failure. Soc Sci Med 2004;58:631643.

11. Granger BB, Swedberg K, Ekman I et al. Adherence to candesartan and placebo and outcomes in chronic heart failure in the CHARM programme: Double-blind, randomised, controlled clinical trial. Lancet 2005;366:20052011.

12. Trojano L, Antonelli Incalzi R, Acanfora D et al. Cognitive impairment: A key feature of congestive heart failure in the elderly. J Neurol 2003;250:1456-1463.

13. Qiu C, Winblad B, Marengoni A et al. Heart failure and risk of dementia and Alzheimer disease: A population-based cohort study. Arch Intern Med 2006;166:1003-1008.

14. Vogels RL, Scheltens P, Schroeder-Tanka JM et al. Cognitive impairment in heart failure: A systematic review of the literature. Eur J Heart Failure 2007;9:440-449.

15. Zuccala G, Onder G, Pedone C et al. Hypotension and cognitive impairment-selective association in patients with heart failure. Neurology 2001;57:1986-1992.

16. Vogels RL, Oosterman JM, van Harten B et al. Profile of cognitive impairment in chronic heart failure. J Am Geriatr Soc 2007;55:1764-1770.

17. Pressler SJ, Subramanian U, Kareken D et al. Cognitive deficits in chronic heart failure. Nurs Res 2010;59:127-139.

18. Juster FT, Suzman R. An overview of the Health and Retirement Study. J Hum Resources 1995;30:S7-S56.

19. Rector TS, Wickstrom SL, Shah M et al. Specificity and sensitivity of claims-based algorithms for identifying members of Medicare plus Choice health plans that have chronic medical conditions. Health Serv Res 2004;39:1839-1857.

20. Birman-Deych E, Waterman AD, Yan Y et al. Accuracy of ICD-9-CM codes for identifying cardiovascular and stroke risk factors. Med Care 2005;43:480-485.

21. Gure TR, McCammon RA, Cigolle CT et al. Predictors of self-report of heart failure in a population-based survey of older adults. Circ Cardiovasc Qual Outcomes 2012;5:396-402.

22. Plassman BL, Newman TT, Welsh KA et al. Properties of the telephone interview for cognitive status - application in epidemiologic and longitudinal studies. Neuropsychiatr Neuropsychol Behav Neurol 1994;7:235-241.

23. Welsh KA, Breitner JCS, Magruderhabib KM. Detection of dementia in the elderly using telephone screening of cognitive status. Neuropsychiatr Neuropsychol Behav Neurol 1993;6:103-110.

24. de Jager CA, Budge MM, Clarke R. Utility of TICS-M for the assessment of cognitive function in older adults. Int J Geriatr Psychiatry 2003;18:318-324.

25. Langa KM, Kabeto MU, Weir D. Percentage of Americans aged 55 and older with selected diseases by race/ethnicity and cognitive status, Health and Retirement Study, 2006. 2010 Alzheimer's Disease Facts and Figures [provided under contract to the Alzheimer's Association], 2010 [on-line]. Available at http://www.alz.org/documents_custom/report_alzfactsfigures 2010.pdf Accessed January 8, 2010.

26. Langa KM, Plassman BL, Wallace RB et al. The Aging, Demographics, and Memory Study: Study design and methods. Neuroepidemiology 2005;25: $181-191$.

27. Crimmins EM, Kim JK, Langa KM et al. Assessment of cognition using surveys and neuropsychological assessment: The Health and Retirement Study and the Aging, Demographics, and Memory Study. J Gerontol B Psychol Sci Soc Sci, 2011;66B(Suppl 1):162-171.

28. Quach S, Blais C, Quan H. Administrative data have high variation in validity for recording heart failure. Can J Cardiol 2010;26:306-312.

29. Shimada SL, Zaslavsky AM, Zaborski LB et al. Market and beneficiary characteristics associated with enrollment in Medicare managed care plans and fee-for-service. Med Care 2009;47:517-523.

30. Plassman BL, Langa KM, Fisher GG et al. Prevalence of dementia in the United States: The Aging, Demographics, and Memory Study. Neuroepidemiology 2007;29:125-132. 